\title{
The effect of 14-day interval on the oscillation effect during sequential alternation of unilateral amygdaloid stimulation
}

\author{
JOHN GAITO \\ York University, 4700 Keele Street, Downsview M3J 1P3, Ontario, Canada
}

\begin{abstract}
Previous research with the kindling paradigm indicated that an oscillation effect resulted during sequential alternation of unilateral amygdaloid stimulation with consistently low values for one side and consistently high values for the contralateral one. The effect of interphase intervals of 1 and 14 days following (Experiment 1) and during (Experiment 2) the development of oscillation was investigated in this study. In Experiment 1 both the 1-day and 14-day intervals disrupted the oscillation tendency in criterion data but not in the latency measure. In Experiment 2 disruption of oscillation occurred only for the criterion measure in the 14-day group.
\end{abstract}

The "kindling effect" has been investigated in a number of laboratories as a model of learning, a model of epilepsy, or as an example of behavioral change of interest in its own right (e.g., Gaito \& Gaito, 1974; Goddard, McIntyre, \& Leech, 1969). This event involves a change from normal exploration (Stage 1, NE), to behavioral automatisms (Stage 2, BA: chewing, eye closure on ipsilateral side, salivation), and finally to clonic convulsions (Stage 3, CC) in response to electrical stimulation of a specific brain site (e.g., the amygdala). Stage 3 behavior involves the rat standing on its hind paws and subsequent bilateral convulsions of the forelimbs. Behavioral, chemical, electrophysiological, and neurological aspects of this effect have been investigated by many researchers (Gaito, 1976a).

If one amygdala (primary site) is stimulated with 50-microA intensity for $5 \mathrm{sec}$ to a criterion (e.g., six CCs), using $24 \mathrm{~h}$ as the intertrial interval, and then the contralateral amygdala (secondary site) is stimulated, the criterion for the latter is reached within a fewer number of trials, but increased latency to convulsion results. Thus, both positive and negative transfer occur (Goddard et al., 1969; McIntyre \& Goddard, 1973). If the animal is stimulated again at the primary site, it does not convulse on the first trial. A few trials are required to reach the $\mathrm{CC}$ stage, thus indicating negative transfer.

Recent research in our laboratory used three trials per day and stimulation at an intensity of 100 microA for $30 \mathrm{sec}$; using a sequence of alternating unilateral stimulations of the amygdala, usually for 10 alternation phases, an "oscillation effect" was observed (Gaito, 1976b). ${ }^{1}$ This oscillation usually involved consistently low values for the amygdala first stimulated (primary site) and consistently high values for the contralateral amygdala (secondary site). The effect was most prominent in latency data (number of seconds from onset of stimulation to onset of convulsion).
A number of variables that might affect this oscillation tendency have been investigated in our laboratory. In latency data, bilateral stimulation prior to, or after, the development of oscillation had no disruptive effect on the oscillation tendency (Gaito, 1976c). The tendency persisted with 1,3 , or 6 trials per day (Gaito, 1976d) and 1,6 , or 12 convulsions per phase (Gaito, 1976e). Oscillation was as prominent in old rats (420-475 days of age) as in younger ones (135-200 days) (Gaito \& Nobrega, 1977). Rest intervals of 1, 3, and 6 months interspersed between two sets of 10 sequential alternation phases had no deleterious effect (Gaito, 1976f). The tendency was unaffected by intensities of 100 and 280 microA, but a 560 -microA intensity had a slight disruptive effect (Gaito, 1977a). Thus, it appears that the oscillation effect is an extremely robust event for latency data.

In the criterion measure (number of trials to a specific number of convulsions, usually six), there were few oscillators. Each of the above variables disrupted partially, or completely, this weak oscillation tendency. The duration of convulsion measure was not analyzed in these experiments because few oscillators were detected in the first study.

Two types of oscillation occur consistently in our research. Primary oscillation (PO) involves low values for the primary site and high values for the secondary site for at least 8 of 10 phases. Secondary oscillation (SO) is the opposite pattern.

With our procedure of alternating stimulations over 10 phases and our requirement that a rat designated as an "oscillator" must show a consistent "low-high" or "high-low" pattern for at least 8 of the 10 phases, the probability that these apparent systematic patterns of oscillation are actually random ones is $.17 .^{2}$ The number of oscillators involved in previous experiments was significantly different from the expected value in every 
experiment in latency data. Thus, this measure appears to be the most sensitive indicator of oscillation, an observation that was obvious from the first study (Gaito, 1976b).

These experiments used 1 day as the interphase interval. McIntyre and Goddard (1973), using sequential alternation over three phases, found an "aftereffect" of negative transfer nature that dissipated in 14 days. The results in our experiments may be due to the McIntyre and Goddard "aftereffect." The present study was concerned with the possibility that the oscillation tendency might be lost with 14-day interphase intervals. The study consisted of two parts: (1) the effects of 14 and 1-day interphase intervals over 10 phases of sequential alternation after rats had developed the oscillation tendency (Experiment 1) and (2) the effects of 14 . and 1-day interphase intervals during the development of kindling over 10 phases of sequential alternation (Experiment 2).

\section{METHODS}

For Experiment 1, 11 male Wistar rats were chosen from previous research; these animals had shown oscillation tendencies in latency data (ages 200-300 days). The rats had been implanted with bipolar electrodes in each amgydala. The brain coordinates for electrode implantation were the same as in many experiments in our laboratory: $.5 \mathrm{~mm}$ posterior to bregma, $4.5 \mathrm{~mm}$ from midline, $8.5 \mathrm{~mm}$ from skull (Gaito \& Gaito, 1974; Gaito, 1976b). The animals were separated into two groups of five and six rats. The side to be stimulated first was determined by a random process. Each rat in both groups received $30 \mathrm{sec}$ of stimulation at an intensity approximately 15 microA above threshold for convulsing using a Lafayette sine-wave stimulator $(60 \mathrm{~Hz})$. The intensities were between 84 and 280 microA for most rats; two animals required 560-microA intensity for one side. Each phase involved stimulation over a number of trials (three per day) until the criterion of six convulsions was attained. The interphase interval for Group 1 was 1 day; for Group 2 it was 14 days.

In. Experiment 2, each of 22 male Wistar rats (approximately 150 days of age) were implanted bilaterally with electrodes in the amygdalae. Stimulation of 100 -microA intensity began 7 days after surgery. With some rats the intensity had to be increased to achieve convulsion during Phases 1 and 2. Threshold values were determined during Phases 3 and 4 and stimulation intensities were set approximately 15 microA above threshold for each rat. The intensities were about the same as in Experi- ment 1. One set of these rats (Group 3, 12 rats) had an interphase interval of 1 day. The interphase interval for the 10 rats in Group 4 was 14 days. Rats were selected randomly for Groups 3 and 4 immediately after the sixth convulsion on Phase 1.

Previous research indicated that similar oscillation tendencies occur if the same intensities are used for both sides or if intensities 15 microA above threshold are used (Gaito, 1977b). However, the latter procedure should eliminate the possibility of differential natural reactivities of the two hemispheres. It is used as a routine procedure in our research.

For each rat the behavioral designation for the latency and criterion measures was that of primary oscillator, secondary oscillator, or nonoscillator. For example, to be classified as an oscillator, the rat had to show a consistent pattern of "low-high" (primary oscillation, PO) or "high-low" (secondary oscillation, SO) in latency and/or criterion data over 8 or 10 of the 10 alternation phases. As in the previous research, duration values were not analyzed.

No histological analyses were performed in either experiment because the analyses of previous research (Gaito, 1976b, 1977b; Nobrega \& Gaito, 1978) indicated no relationship between electrode placement and behavioral pattern, as long as one or both electrodes were in the amygdala or in nearby structures.

\section{RESULTS}

The results for Experiment 1 are shown in Table 1. For Group 1, three rats had oscillated in the criterion measure of the previous research (two POs, low values from primary site and high values for secondary site, and one SO, low values for secondary site and high values for primary site) and two were nonoscillators; no oscillation occurred in the present experiment. In the latency measure, all rats had oscillated previously (three POs, two SOs). Four of the five rats continued to oscillate.

Of the six rats in Group 2, four had oscillated in the previous research (all SOs) in the criterion data. None oscillated in Experiment 1. For latency data, all had oscillated previously (three POs, three SOs). Five of the six rats continued with the same oscillation pattern in Experiment 1.

Thus, oscillation terminated in the criterion measure for both groups. Not only is the criterion measure a weak indicator of oscillation (as indicated in all previous research), it is also an unstable one.

Table 1

Pattern of Behavior of Rats in Experiment 1

\begin{tabular}{|c|c|c|c|c|c|c|c|c|c|}
\hline \multicolumn{5}{|c|}{ Group 1 (1 day) } & \multicolumn{5}{|c|}{ Group 2 (14 days) } \\
\hline \multirow[b]{2}{*}{ Rat } & \multicolumn{2}{|c|}{ Criterion } & \multicolumn{2}{|c|}{ Latency } & \multirow[b]{2}{*}{ Rat } & \multicolumn{2}{|c|}{ Criterion } & \multicolumn{2}{|c|}{ Latency } \\
\hline & Previous & Present & Previous & Present & & Previous & Present & Previous & Present \\
\hline 1 & NO & NO & PO & NO & 2 & so & NO & SO & so \\
\hline 3 & PO & NO & PO & PO & 4 & so & NO & PO & NO \\
\hline 5 & PO & NO & PO & PO & 6 & NO & No & PO & PO \\
\hline 7 & so & NO & so & so & 8 & so & NO & so & so \\
\hline \multirow[t]{2}{*}{9} & NO & No & so & so & 10 & NO & NO & PO & PO \\
\hline & & & & & 11 & so & NO & so & so \\
\hline
\end{tabular}

Wote $-P O=$ primary oscillation $; S O=$ secondary oscillation; $N O=$ nonoscillation. 
Table 2

Pattern of Behavior of Rats in Experiment 2

\begin{tabular}{|c|c|c|c|c|c|}
\hline \multicolumn{3}{|c|}{ Group 3 (1 day) } & \multicolumn{3}{|c|}{ Group 4 (14 days) } \\
\hline Rat & Criterion & Latency & Rat & Criterion & Latency \\
\hline \multirow{14}{*}{$\begin{array}{r}1 \\
3 \\
5 \\
7 \\
9 \\
11 \\
13 \\
15 \\
17 \\
19 \\
21 \\
22\end{array}$} & PO & PO & 2 & NO & PO \\
\hline & PO & PO & 4 & NO & PO \\
\hline & NO & NO & 6 & NO & NO \\
\hline & so & PO & 8 & NO & PO \\
\hline & So & NO & 10 & NO & SO \\
\hline & SO & NO & 12 & NO & PO \\
\hline & PO & PO & 14 & NO & So \\
\hline & NO & NO & $16^{*}$ & NO & NO \\
\hline & NO & SO & 18 & NO & NO \\
\hline & PO & SO & 20 & NO & NO \\
\hline & $\begin{array}{l}\text { NO } \\
\text { PO }\end{array}$ & $\begin{array}{l}\text { PO } \\
\text { PO }\end{array}$ & & & \\
\hline & 5 POs & 6 POs & & 10 NOs & 4 POs \\
\hline & $3 \mathrm{SOs}$ & $2 \mathrm{sOs}$ & & & 2 SOs \\
\hline & 4 NOs & 4 NOs & & & 4 NOs \\
\hline
\end{tabular}

*Lost electrode after six phases.

The latency data provided both a strong and stable indicator of oscillation. Neither the 1-nor the 14-day interphase intervals disrupted the oscillation tendency; four of five rats in Group 1, and five of six in Group 2, persisted in the same oscillation pattern as in previous research.

In Experiment 2, 8 of the 12 rats in Group 3 (1-day interval) showed oscillation behavior in criterion and in latency data, with primary oscillation predominating in both cases (Table 2). For the 14-day groups, none of the 10 rats oscillated in the criterion measure, but 6 of 10 oscillated in latency data. Based on .17 as the probability of oscillation, 1.7 and 2 rats would be expected to oscillate for 10 and 12 rats, respectively. The probability that no rats will oscillate is .155 (using the binomial distribution) and, thus, this number is not significantly different from the number expected. However, the 6 rats oscillating in the sample of 10 rats in latency data for the 14-day group, and the 8 rats oscillating of the 12 rats in latency and criterion data for the 1-day group, were each significantly different from the number of oscillations expected ( $p$ well below .01).

In the first study (Gaito, 1976b), the mean duration values were greater for rats that were rested for 17 to 21 days between stimulation phases than for rats that were stimulated each day. In Experiments 1 and 2, the duration values were compared for the 1-and 14-day groups (Table 3). The values for Groups 1 and 3 (1-day intervals) were similar to those observed in previous research, in general varying between 20 and $30 \mathrm{sec}$. However, the mean values for the two 14-day groups tended to be greater than those for the 1-day groups after the first phase. The first phase should produce similar values, because the 14- and 1-day interphase interval is not introduced until Phase 1 is complete. In all nine phases, the mean duration values were greater for the 14-day groups. The discrepancy between the 1 and 14 days was small on Phase 2 but increased drastically thereafter; usually there was a $10-$ to $20-\mathrm{sec}$ difference.

\section{DISCUSSION}

The conclusion from these experiments is that, under our stimulating conditions, the oscillation tendency is a robust and stable phenomenon in latency data. As in previous experiments, the oscillation tendency was more prominent in latency data than in the criterion measure. In Experiment 1 both the 1- and 14-day intervals produced no oscillation tendency in criterion data but did in the latency measure. In Experiment 2 disruption of oscillation occurred only for the criterion measure in the 14-day group.

Although a significant number of oscillators seldom appear in criterion data, in Experiment 2 the oscillation tendency was as strong in these data as in the latency measure with the 1-day interphase interval. However, the 14-day interval appeared to disrupt the development of the oscillation tendency in criterion data, in that no rats oscillated. These results are consistent with the hypothesis that the McIntyre and Goddard (1973) "aftereffect" was responsible for the consistent differences in primary and secondary site values for oscillating rats.

On the other hand, the latency results are not consistent with the aftereffect hypothesis in that the oscillation tendency was present for both the 1- and 14-day groups in Experiment 2. However, it is possible that a type of aftereffect operates in the oscillation process, but at a more potent level than in the McIntyre and Goddard experiments, because of different stimulating conditions. McIntyre and Goddard used 50-microA intensity for $5 \mathrm{sec}$ with one trial per day for most of their rats. We used 100 microA or more for $30 \mathrm{sec}$ over three trials each day for most rats. Thus, the total daily energy involved with our stimulating conditions was much greater than was the case for the rats in the McIntyre-Goddard study.

The duration results in these experiments were similar to those in the first study also. Mean duration values tended to be 10 to $20 \mathrm{sec}$ greater after Phase 1 for the rats rested for 14 days between stimulation phases than for those that had a 1-day interphase interval. Presumably, with time, a sensitivity condition develops in brain tissue such that the convulsion continues for a longer period of time. The longest duration in our laboratory over approximately 5 years of kindling research was for a

Table 3

Mean Duration Values (in Seconds)

\begin{tabular}{cccccccccccc}
\hline & & \multicolumn{10}{c}{ Phases } \\
\cline { 2 - 11 } & Group & 1 & 2 & 3 & 4 & 5 & 6 & 7 & 8 & 9 & 10 \\
\hline \multirow{2}{*}{ Experiment 1 } & 1 & 33.6 & 22.0 & 24.8 & 24.0 & 24.8 & 23.0 & 21.5 & 20.7 & 24.9 & 17.5 \\
& 2 & 30.3 & 26.7 & 33.4 & 35.4 & 30.6 & 36.1 & 37.8 & 36.8 & 39.1 & 37.6 \\
\multirow{2}{*}{ Experiment 2 } & 3 & 23.5 & 25.4 & 21.9 & 24.7 & 25.3 & 27.2 & 23.7 & 29.6 & 18.6 & 25.7 \\
& 4 & 22.6 & 27.0 & 35.5 & 34.3 & 40.3 & 38.7 & 39.4 & 37.2 & 39.8 & 40.0 \\
\hline
\end{tabular}


rat that convulsed for $4 \frac{1}{2} \mathrm{~min}$; the rat had rested for 30 days. Although oscillation is a frequent pattern of behavior over 10 or more phases of sequential alternation stimulation, the basis for the pattern is unclear. Presumably, there are some inhibitory and/or facilitatory effects from the primary site to the secondary site, and vice versa, to produce the oscillation effect (Nobrega \& Gaito, 1978). An oscillation pattern appears to be set up during Phases 1 and 2, or by Phases 3 and 4, and most rats continue the pattern over all phases, or over many phases, before it extinguishes.

The unilateral sequential alternation procedure and the resulting oscillation effect appear to be useful for obtaining information on some aspects of the events underlying the kindling effect (e.g., possible differential electrical synaptic patterns related to the difference in latency values for the primary and secondary sites). Because kindling shows many parallels to learning and to epilepsy, and can be considered as a model of consistent behavioral changes in response to an invariant stimulus (Gaito, 1976a), experimentation with the oscillation effect may have the potential for providing information relevant to brain function in general.

\section{REFERENCES}

GaIto, J. The kindling effect as a model of epilepsy. Psychological Bulletin, 1976, 83, 1097-1109. (a)

GAITo, J. An oscillation effect during sequential alternations of unilateral amygdaloid stimulations with the kindling paradigm. Physiological Psychology, 1976, 4, 303-306. (b)

GaITo, J. The effect of bilateral stimulation during sequential alternation of unilateral amygdaloid stimulation. Bulletin of the Psychonomic Society, 1976, 4, 355-357. (c)

GaITo, J. The effect of number of trials per day during sequential alternation of unilateral amygdaloid stimulation. Bulletin of the Psychonomic Society, 1976, 4, 403-404. (d)

GaIto, J. The effect of number of convulsions per phase on the oscillation tendency. Bulletin of the Psychonomic Society, 1976, 8, 392-394. (e)

GaITo, J. The effect of varying rest intervals following the development of oscillation during unilateral amygdaloid stimulation. Bulletin of the Psychonomic Society, 1976, 8, 457-458. (f)

Garto, J. The effect of intensity during sequential alternation of unilateral amygdaloid stimulation. Bulletin of the Psychonomic Society, 1977, 9, 64-66. (a)

GaITo, J. The oscillation effect at near threshold intensities during sequential alternation of unilateral amygdaloid stimulation. Bulletin of the Psychonomic Society, 1977, 10, 145-148. (b)

GaITo, J., \& GAITo, S. T. Interanimal negative transfer of the kindling effect. Physiological Psychology, 1974, 2, 379-382.

Gaito, J., \& Nobrega, J. The oscillation effect during sequential alternation of amygdaloid stimulation with aged rats. Bulletin of the Psychonomic Society, 1977, 9, 151-154.

Gaito, J., Nobrega, J. N., \& GaIto, S. T. Statistical evaluation of several aspects concerning the oscillation effect.. Physiological Psychology, in press.

Goddard, G. V., McIntyre, D. C., \& Leech, C. K. A permanent change in brain function resulting from daily electrical stimulation. Experimental Neurology, 1969, 25, 295-330,

HoEL, P. G. Introduction to mathematical statistics. New York: Wiley, 1954.

MCINTYRE, D. C., \& GoddARD, G. V. Transfer, interference and spontaneous recovery of convulsions kindled from the rat amygdala. Electroencephalography and Clinical Neurophysiology, 1973, 35, 533-543.

NobreGA, J. N., \& Garto, J. Long term induction of kindled seizures in rats: Interhemispheric factors. Canadian Journal of Neurological Sciences, 1978, 5, 223-230.

\section{NOTES}

1. This term is based on the curve obtained from plotting mean latency values over 10 phases; a saw-tooth oscillationtype figure occurs.

2. The hypothesis that the oscillation patterns are random can be assessed by the one-sample runs test (Hoel, 1954). Using the requirement of 8 or 10 phases of oscillation out of 10 phases provides 8,9 , or 10 runs. The $p$ of 8,9 , and 10 runs is $.1270, .0317$, and .0079 , respectively; the $p$ of 8,9 , or 10 runs is the sum of these ps, .1666 or .17. Thus, the p that these apparent systematic patterns are random would be .17 (Gaito, Nobrega, \& Gaito, in press).

(Received for publication April 18, 1978.) 